\title{
Optimizing thymic recovery in HIV patients through multidrug therapies
}

\author{
V. Costanza ${ }^{1, \sharp, \dagger}$, P. S. Rivadeneira $a^{\sharp}$, F. L. Biafore ${ }^{\ddagger}$, C. E. D’Attellis ${ }^{\dagger, \ddagger}$ \\ ${ }^{\dagger}$ Centro de Matemática Aplicada, Escuela de Ciencia y Tecnología, UNSAM \\ M. de Irigoyen 3100, 1650 San Martín, Pcia. de Buenos Aires, Argentina \\ ${ }^{\ddagger}$ Grupo de Ingeniería Biomédica, Facultad de Ingeniería, Universidad Favaloro \\ Avda. Belgrano 1723, 1093 Buenos Aires, Argentina \\ ${ }^{\sharp}$ Grupo de Sistemas No Lineales, INTEC(UNL-CONICET) \\ Güemes 3450, 3000 Santa Fe, Argentina
}

\begin{abstract}
An enlarged nonlinear model for the dynamics of HIV infection and thymic function is composed to simulate and evaluate antiretroviral therapies. In addition to the relevant biological agents, an extra state variable is included, associated with the thymus capacity for healthy cells production. The methodology contemplates eventual deleterious effects of drugs over children's thymus recovery. The intake of RTI and PI drugs are modelled as two independent control variables, each affecting a different term in the dynamics, so extending the prevailing pure-HAART-therapy analysis. The objective function designed here is also more inclusive than usual, accounting for the costs of the two drug families involved and for the thymus deterioration, in addition to penalizing eventual virus excess and healthy cells deficits. The search for the best combined therapy is treated as an optimal control problem. An hybrid version of Dynamic Programming for continuous and discrete variables is used to treat the problem numerically. Long time-horizons are explored, aiming to avoid typical peaks in drug prescriptions found at the beginning and end of the optimization periods. Results indicate that certain combinations of drugs are more convenient than pure protocols when the value of thymus functioning is relevant, specially for children patients.

Keywords

HIV, Antiretroviral Drugs, Optimal Control, Nonlinear Dynamics, Dynamic Programming.
\end{abstract}

\section{Introduction}

The role of the thymus in the regeneration of healthy CD4+ T cells during HIV infection has been widely studied in the last decade (see [11], [12] and the references therein). It is likely that such a regenerative property is more relevant in children [9], [10], although in all cases it is accepted that HIV presence deteriorates thymus functioning, and therefore that the thymopoiesis at a nearly constant rate can not be assured after the appearance of virus, specially in its

\footnotetext{
${ }^{1}$ Corresponding author.
} 
CXCR4 version [12]. The negative effect of HIV on thymus functioning is partially reversed by the HAART therapy [3], [28], and it is also found that the later the therapy is initiated the slower the recuperation of normal behavior will be achieved [26]. But HAART has shown to produce severe side-effects (see [6] and its references) so it is worth to search for milder medications that still reduce the viral load to protocol target values.

Even in the absence of virus, the thymus is one of the first organs to undergo significant age-related degeneration, termed thymic involution. Thymic involution results in a dramatic drop in the production of new T-cells, and is a significant contributing factor in immune senescence. Despite the importance of this subject for human health, the molecular dynamics that operate in the postnatal thymus and mediate thymic homeostasis and involution are largely unknown [16]. Detailed mathematical models have been developed at cellular level [14], [28], aiming to reflect all relevant interactions affecting the intervening agents, to the cost of including numerous state variables, most of them difficult to measure (or to observe) continuously. According to applicable findings of these studies, the dynamical models used by the authors in previous publications [5], [6] were modified to include variations on healthy cells' regeneration rate and thymus function decay. For instance, it has turned clear that the parameter associated with the thymopoiesis in simple models should become a state variable in itself, reflecting thymic function dynamics. The new variable declines in the presence of HIV infection [24], and this affects the immune response negatively, most in the from of a self-feeding loop. It is known that recovery of thymic function could occur in HIV-1-infected patients on HAART. However, specific mechanisms that contribute to this recovery are still under study ([28], [17]).

Also it is now widely accepted that dynamic models should account for differences between children and adults' evolution of the infection and thymic function, and also in their respective responses to medication [21], [24], [28], [9]. In this last respect, this paper will emphasize the importance of evaluating alternative drug families for medication, since their affecting different terms or parameters of the model will provide alternatives to standard protocols. The evaluation of these alternatives will contemplate viral load suppression, thymic function recovery, and medication expenses, giving rise to a combined cost functional with competing individual objectives. The relative importance of these individual objectives into the total cost, and the addition or supression of other objectives, has been discussed in international forums [27].

The model for the dynamics together with the cost functional designed for therapies evaluation pose an optimal control problem. This problem will adopt a richer viewpoint in comparison with most approaches in previous papers, since innovations are included in the model and in the evaluation functional, both containing more variables and individual cost objectives. A special feature in this last respect will be the consideration of long optimization time-horizons, in the order of several years, in an attempt to avoid big variations in the drug doses typically prescribed at the beginning and the end of shorter time-horizons (of around 180 days) [6]. Another improvement in the scope of the optimization 
problem is the possibility of including two families of drugs into the medication, eventually in variable proportions. The doses admitted here will contain typical members of the Reverse Transcriptase Inhibitors (RTI) and the protease Inhibitors (PI) groups.

The results arising from the numerical treatment of this optimization setup support two main conclusions: (i) a combination of RTI and PI drugs can work better than monodrug protocols when all individual cost objectives are given proper weight, and (ii) less aggressive drugs can partially replace HAART during small infected children medication, especially when avoiding thymus dysfunctionality is assigned a significant value.

From the numerical simulations there arise other observations, whose precise meaning deserve further investigation. For instance, it seems that extending the time-horizon in optimization analyses may conduct to reliable therapies consisting of a constant dose for each intervening drug. This outcome is positive in avoiding big changes in therapy typically appearing at the beginning and end of optimization periods of the order of 180 days. But the optimal required doses become higher the longer the horizon is posed, which is not neatly positive in several respects, not the least the increasing money-cost of the medication. A first interpretation would propose that higher doses are required to give more assurance against predictable rebounds of the viral load. However, it is also true that side-effects of higher doses will increase, and that should also be included as a partial cost in the optimization analysis, as done in previous work [6]. Unwanted effects of medication were not considered in this paper due to lack of data concerning both combined therapies and long intervals of application.

The paper is organized as follows: after the Introduction there is a Section explaining the model for the dynamics of the control system under study, and in Section 3 the cost functional is designed and discussed. Section 4 gives the details of the calculation techniques used to find optimal therapies, and the numerical results obtained are analyzed in Section 5. The final Section summarizes the conclusions of the paper.

\section{Dynamics of infection and thymus function}

The behavior of infected patients will be modeled through a continuous-time control system described by the following set of coupled ordinary differential equations:

$$
\begin{aligned}
& \dot{x}=\lambda-\delta x-\beta\left(u_{1}\right) x z \\
& \dot{y}=\beta\left(u_{1}\right) x z-\mu y \\
& \dot{z}=\kappa\left(u_{2}\right) y-\gamma z \\
& \dot{\lambda}=\eta\left(u_{1}, u_{2}\right)\left(\lambda_{m}-\lambda\right)-\sigma z
\end{aligned}
$$

where the states are: healthy CD4+ T cells $(x)$, infected CD4 T cells $(y)$, free virus copies or virions $(z)$, and thymopoiesis rate $(\lambda)$.

The control action over the states is exerted by the medication, i.e. by the intake of drugs. Basically, the antiretroviral drugs can be grouped into the following three categories ([13]): 
(i) Inhibitors of the reverse transcriptase enzyme (RTIs): If RT is inhibited, HIV can enter a cell but will not successfully infect it; a DNA copy of the viral genome will not be made and the cell will not make viral proteins.

(ii) Protease inhibitors (PIs): If HIV protease is inhibited, cleavage of the viral polyprotein will not occur, and viral particles that lack functional enzymes will be made. The net effect of blocking HIV protease is that noninfectious viral particles are made.

(iii) Fusion Inhibitors (FIs): these work by inhibiting the binding of HIV to healthy CD4+T-cells (used in patients with multi-drug HIV resistance, not studied in this paper).

The control variables will be restricted in this paper to $u_{1}$ : dose of Zidovudine AZT (RTI), and $u_{2}$ : dose of Ritonavir (PI), both in [g/day] .

The first three equations (when $\lambda, \beta\left(u_{1}\right)$ and $\kappa\left(u_{2}\right)$ are kept constant, as if they were parameters) constitute a classical simplified model [18], [19], [5], thus further comments on the meaning of their terms seem unnecessary at this time. The last equation for $\lambda$ dynamics is new. It governs the natural thymus homeostasis. No data have been reported describing the ability of HAART agents to cross the blood-thymus barrier and penetrate into the thymus. Therefore the explicit dependence of the thymus variable $\lambda$ on any of the control variables $u_{i}$ adopted in the last of Eqs. (1) should be designed as to give a reasonable approximation to the observed qualitative behavior reported up to now. The form adopted here includes

$$
\eta\left(u_{1}, u_{2}\right) \triangleq\left[1-\chi u_{1}\left(1-u_{2}\right)\right]
$$

where $\chi$ is a nonnegative parameter. Supposing there is no infection for the moment $\left(z=0\right.$, and consequently with no medication, i.e. $u_{1}=u_{2}=0, \eta\left(u_{1}, u_{2}\right)=$ 1 ), and if the term $\left(\lambda_{m}-\lambda\right)$ is positive (the thymus functioning is below its standard value $\lambda_{m}$ ), then a self-induced recuperation will be intended by the human organism, i.e. the rate $\dot{\lambda}=d \lambda / d t$ will increase. But the HIV infection, reflected in the present value of the state $z(t)>0$, will oppose this recovering through the term $-\sigma z, \sigma$ a parameter identified from: (i) the available data for thymus dynamics showing, for instance, that the thymocytes decay to about one half of their original number [28], [14] during the first month after infection, together with (ii) the conventionally accepted proportional relation between thymocytes and CD4+ T cells. The behavior of $\eta\left(u_{1}, u_{2}\right)$ in the range of interest of the control doses can be seen in Fig. 1. Setting

$$
\chi_{\text {adults }}=0
$$

implies $\eta_{\text {adults }}\left(u_{1}, u_{2}\right) \equiv 1$ for any admissible combination of RTI and PI drugs, coincident with the observation that for adults the thymus recovery seems to be independent of drug efficiency inside the organ [14].

Children have a better production of naive T-cells and thymocytes, which suggests, in terms of parameters,

$$
\lambda_{m_{\text {children }}}>\lambda_{m_{\text {adults }}}
$$




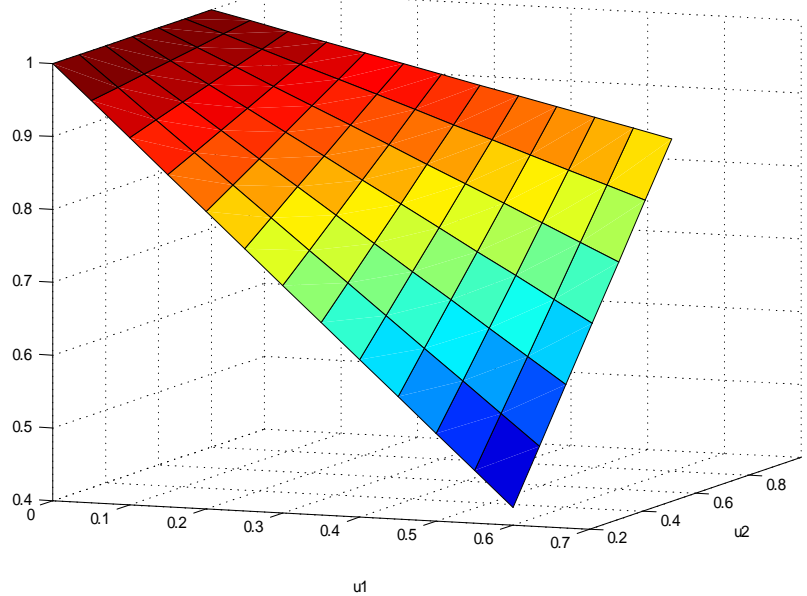

Figure 1: Function $\eta\left(u_{1}, u_{2}\right)=\left[1-\chi u_{1}\left(1-u_{2}\right)\right]$ acting as a coefficient for the recuperation term $\left(\lambda_{m}-\lambda\right)$ in the dynamic equation for the thymic function. Here $\chi=\chi_{\text {children }}=1.2$.

But also the efficiency of drugs inside the thymus is different for children and adults. In previous work we have only considered the action of RTIs. But if only RTI drugs are being dispensed to children patients, it is found that their efficiency inside the thymus is low [28], i.e. such a medication is not enough to produce the same thymus recovery effect than in adults (this is the explanation for $\eta_{\text {children }}\left(u_{1}, u_{2}\right)<1$ for $u_{1}>0$, Eq. (2) and Fig. 1). The same authors indicate that the combination with PI drugs enhances the performance of RTI medication, and that is the reason for the term $\left(1-u_{2}\right)$ multiplying $u_{1}$ in Eq. (2). The combination $\chi u_{1}\left(1-u_{2}\right)$ works as a typical interactive term between the two drugs: $\left(1-u_{2}\right)<1$ reduces the detrimental action due to $u_{1}$ alone. In order to keep $0<\eta_{\text {children }}\left(u_{1}, u_{2}\right) \leq 1$ in the range of interest, the adopted value for the relevant parameter was

$$
\chi_{\text {children }}=1.2 \text {. }
$$

The asymptotic value for the thymus function of an infected non medicated patient $\left(u_{1}=u_{2} \equiv 0\right)$ will be

$$
\bar{\lambda} \triangleq \lambda_{m}-\frac{\sigma}{\bar{z}},
$$

where the symbol $\bar{z}$ represents the 'ill' equilibrium value of the viral load. The point $(\bar{x}, \bar{y}, \bar{z})=\left(\frac{\mu \gamma}{\beta \kappa}, \frac{\lambda-\delta \bar{x}}{\mu}, \frac{\kappa \bar{y}}{\gamma}\right) \in \mathbb{R}^{3}$ describes the values approached by the first three state variables when the patient is critically infected and non medicated At such values the dynamics would become asymptotically stable, 
tending to a terminal situation with $\dot{x}=\dot{y}=\dot{z}=0$. By assuming that $\bar{z}$ is approximately the same for children and for adults, the fact that the thymus function of infected children is more susceptible of becoming dysfunctional [4], [10] will be interpreted as the need for

$$
\bar{\lambda}_{\text {children }}<\bar{\lambda}_{\text {adults }},
$$

which in view of Eqs. (4-6) would only be possible provided that also

$$
\sigma_{\text {children }}>\sigma_{\text {adults }} \text {. }
$$

The remaining nominal values for the parameters in Eqs. (1) are then recovered from previous versions of the model and fitted to meet the differences between children and adults discussed in the text and citations above:

$$
\begin{aligned}
& \lambda_{m_{\text {adults }}}=9 \text { cells } \mathrm{mm}^{-3} \text { day }^{-1} \\
& \lambda_{m_{\text {children }}}=9.5 \text { cells mm }^{-3} \text { day }^{-1} \\
& \sigma_{\text {adults }}=2 \times 10^{-5} \text { cells copies }^{-1} \mathrm{~mm}^{-3} \text { day }^{-2} \\
& \sigma_{\text {children }}=6 \times 10^{-5} \text { cells copies }{ }^{-1} \mathrm{~mm}^{-3} \text { day }^{-2} \\
& \delta=0.009 \text { day }^{-1} \\
& \mu=0.3 \text { day }^{-1} \\
& \gamma=0.6 \text { day }^{-1}
\end{aligned}
$$

The proposed expression for $\beta\left(u_{1}\right)$

$$
\beta\left(u_{1}\right) \approx \beta_{0}-\alpha_{1} u_{1}-\alpha_{2} u_{1}^{2}
$$

was substantiated from qualitative and qualitative data in [1], [22], [25]. The parameters were estimated through standard least-squares regression techniques, by assuming that for a short period after therapy has begun, $x$ remains approximately constant (say $x(t) \approx \tilde{x} \triangleq x(0)$ ). Then, the equations for $\dot{y}$ and $\dot{z}$ in the model (1) become linear and their solution imply:

$$
z(t) \approx\left(k_{1} e^{a_{1} t}+k_{2} e^{a_{2} t}\right) z(0),
$$

with coefficients depending on $x(0), y(0), \beta_{0}, \alpha_{1}, \alpha_{2}$. Data in the quoted literature, actually corresponding to treatments of recently discovered infections, produce the following estimates

$$
\begin{aligned}
& \beta_{0}=4 \times 10^{-6} \mathrm{ml} \operatorname{copies}^{-1} \mathrm{day}^{-1}, \\
& \alpha_{1}=0.88 \times 10^{-6} \\
& \alpha_{2}=0.3 \times 10^{-6} .
\end{aligned}
$$

Analogously, PI drugs are known to affect preferentially the pseudo parameter $\kappa$ in a form which has also been adapted to empirical data [2], [20], [23] to obtain:

$$
\kappa\left(u_{2}\right) \approx \kappa_{0}-\alpha_{3} u_{2}-\alpha_{4} u_{2}^{2}
$$




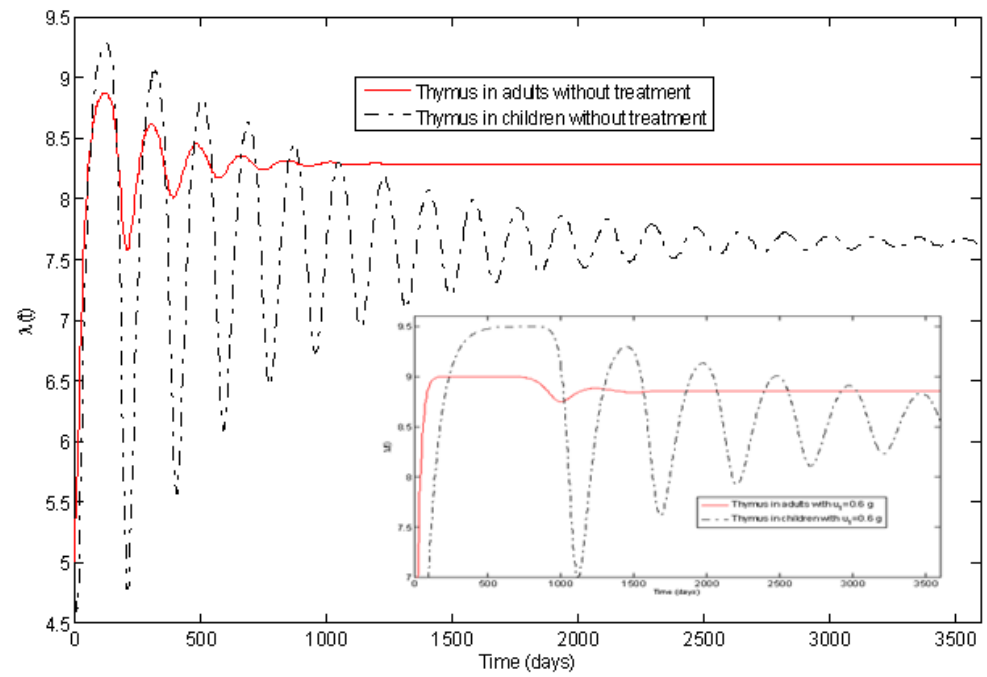

Figure 2: Asymptotic behavior of thymus function for infected children and adults: (i) without medication, and (ii) under a constant pure RTI therapy in the smaller window. Initial conditions as in Eq. (18).

$$
\begin{aligned}
& \kappa_{0}=8 \times 10^{-2} \text { copies cells }^{-1} \mathrm{day}^{-1} \\
& \alpha_{3}=40.943 \\
& \alpha_{4}=1.589
\end{aligned}
$$

Some aspects of the behavior of thymus function variable $\lambda$ for children and adults, in concordance with the comments above, can be visualized in Figures 2 and $3 . \quad$ Figure 2 also shows that, from a lower value $\lambda(0)$ showing some deterioration of the thymus function due to infection, its recuperation in children is obtained after stronger oscillations than in adults, most probably due to the children's greater thymus volume, which allows for a quick response giving rise to more abundant thymocytes than adults do, i.e. generating comparatively more CD4+ $\mathrm{T}$ cells, able to be infected and reproducing more virus, which in turn deteriorate thymus more significantly, and starting the recuperation cycle again. Perhaps this strong oscillations explain early thymus dysfunctionality in children when not properly medicated. Figure 3 shows that combined RTI plus PI therapies could restore thymus function in children to a higher value and more smoothly than with pure RTI drugs. The question is what the best proportion for such combinations is when taking a multi-objective viewpoint. This will be attempted in the rest of the paper. 


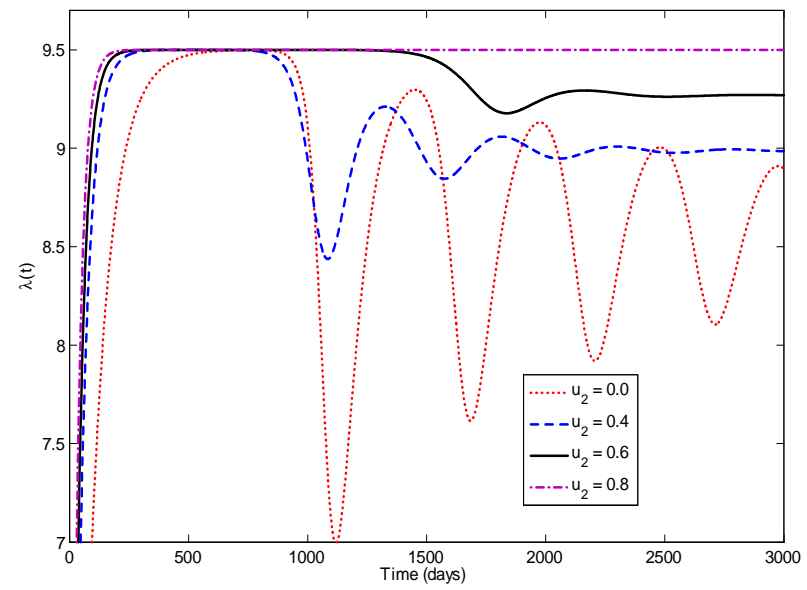

Figure 3: Asymptotic behavior of thymus function for infected children and adults with several constant PI therapies in addition to a constant RTI dosis $u_{1} \equiv 0.6$. Initial conditions as in Eq. (18).

\section{Total cost associated to a therapeutic strategy}

A typical objective functional, representing the "total" cost to minimize amongst all acceptable therapies, may be designed as follows

$$
\begin{aligned}
J(u) & =Q\left(t_{0}, T, x_{0}, y_{0}, z_{0}, \lambda_{0}, u\right)+K(x, y, z, \lambda)_{T} \\
& =\int_{t_{0}}^{T}\left[a_{1} z(t)+a_{2} f\left(u_{1}(t), u_{2}(t)\right)+a_{3}\left(\lambda(t)-\lambda_{m}\right)^{2}\right] d t+a_{4} z^{2}(T) .
\end{aligned}
$$

This is a typical form for the objective function, consisting of a "trajectory cost" $Q\left(t_{0}, T, x_{0}, y_{0}, z_{0}, \lambda_{0}, u\right)$ (usually expressed as the integral of the Lagrangian function $L(x, y, z, \lambda, u)$, which models the cost differentials occurring during treatment), and a "final penalty" $K(x, y, z, \lambda)_{T}$ associated with the departure of the final states from the desired (target) after therapy, which has been maintained quadratic (as in previous work of the authors, for eventual comparison of results), i.e.

$$
K(x, y, z, \lambda)_{T}=K(x(T), y(T), z(T), \lambda(T))=a_{4} z^{2}(T) .
$$

The term $a_{2} f\left(u_{1}, u_{2}\right)$ associated with the cost of the drugs used for therapy should be zero if medication is absent $\left(u_{1}=u_{1}=0\right)$. If there were no other (positive) terms in the trajectory cost $Q$ (see for instance [15] and some of the 
references therein), it may happen that the optimal policy result "no medication". Adding state-trajectory costs ( $a_{1}$ and $a_{3}$ not simultaneously null) will prevent from total drug interruption at any intermediate time within optimal therapies. Such interruptions are recognizedly inappropriate [7]. The choice made here takes the form

$$
L(x(t), y(t), z(t), \lambda(t), u(t))=a_{1} z(t)+a_{2} f\left(u_{1}(t), u_{2}(t)\right)+a_{3}\left(\lambda(t)-\lambda_{m}\right)^{2}
$$

The Lagrangian in this case includes the term $a_{1} z(t)$ penalizing the existing of a nontrivial virus load. The values of $a_{1}$ and $a_{4}$,although adjustable as all evaluation parameters, have been kept high enough as to guarantee that the viral load descend below the 50 copies/ml in the first 180 days for every admissible optimal medication strategy.

The term $f\left(u_{1}, u_{2}\right)$ represents the effective cost of the doses prescribed by the chosen therapy. The simplest form for the function $f$ is just the sum of the individual costs of each drug, i.e.

$$
f\left(u_{1}, u_{2}\right)=m_{1} u_{1}+m_{2} u_{2},
$$

with $m_{i}$ denoting the current price of drug $u_{i}$ in dollars per gram (in Argentina, March 2011).

The coefficient $a_{3}$ wights the importance given to thymus deterioration in the whole. The square in $\left(\lambda-\lambda_{m}\right)^{2}$ reflects the current presumption [24] that a too active thymus is also detrimental in curing the infection, above all in children.

The initial time $t_{0}$ is taken as zero since both the dynamics and the cost functional are autonomous. Then the final time $T$ becomes the time-horizon for the problem. A value of $T \gg 180$ days was used in all calculations, in an attempt to find stable therapies and to avoid typical high doses at the beginning and end of short successive optimization periods (in the order of 180 days).

The values of the coefficients adopted after previous considerations were:

$$
\begin{aligned}
a_{1} & =0.01 \\
a_{2} & =1 \\
a_{3_{\text {children }}} & =100 \\
a_{3_{\text {adults }}} & =50 \\
a_{4} & =0.001 \\
m_{1} & =15.4{\text { dollars } \mathrm{g}^{-1}} \\
m_{2} & =21.6{\text { dollars } \mathrm{g}^{-1}} \\
T & =420 \rightarrow 1200 \text { days }
\end{aligned}
$$




\section{Numerical treatment}

\subsection{Discretizing the state and control spaces}

The structure of a continuous-time optimal control problem is not completely functional to the handling of HIV medication in real life. Notwithstanding the patient's health undergoes a continuos deterioration, possibly following a model like Equations (1), the assessment of the situation can only be made through periodic (discontinuous) blood analyses and doctor inspections, and the present administration of drugs can only be made through discrete amounts and changed every some time-period according to prescriptions. This means that, even when the system evolves in continuous time and the cost objective can theoretically be posed in the same context, the discrete nature of:

(i) measurements' availability or observability of the state variables $(x, y, z, \lambda)$,

(ii) the discrete nature of admissible control values,

(iii) the existence of restrictions in the admissible control values, which hinders the smoothness of control trajectories, and

(iv) the usual delay between physician interventions and control decisions,

it is reasonable to consider a mixed continuous/discrete approach to the numerical treatment.

In what follows, the values of the states and control variables will be then discretized according to the following scheme

$$
\begin{aligned}
\mathcal{X} & \triangleq\left\{x_{L}, x_{L}+\Delta x, x_{L}+2 \Delta x, \cdots, x_{U}\right\} \\
\mathcal{Y} & \triangleq\left\{y_{L}, y_{L}+\Delta y, y_{L}+2 \Delta y, \cdots, y_{U}\right\} \\
\mathcal{Z} & \triangleq\left\{z_{L}, z_{L}+\Delta z, z_{L}+2 \Delta z, \cdots, z_{U}\right\} \\
\mathcal{L} & \triangleq\left\{\lambda_{L}, \lambda_{L}+\Delta \lambda, \lambda_{L}+2 \Delta \lambda, \cdots, \lambda_{U}\right\} \\
\mathcal{U}_{1} & \triangleq\left\{u_{1_{L}}, u_{1_{L}}+\Delta u_{1}, u_{1_{L}}+2 \Delta u_{1}, \cdots, u_{1_{U}}\right\} \\
\mathcal{U}_{2} & \triangleq\left\{u_{2_{L}}, u_{2_{L}}+\Delta u_{2}, u_{2_{L}}+2 \Delta u_{2}, \cdots, u_{2_{U}}\right\} \\
\mathcal{U} & \triangleq \mathcal{U}_{1} \times \mathcal{U}_{2}
\end{aligned}
$$

where the values of the lower $\left.{ }_{L}\right)$ and upper $\left(_{U}\right)$ bounds and the grid size $(\Delta)$ for each variable should depend on real constrains on the appreciation of measurement devices, the possibilities of dose subdivision, calculation capabilities, and expectations. However it is important to note that the adopted values for $y_{L}$, $z_{L}$ and $p_{L}$ should be strictly greater than zero to avoid an unrealistic stagnation of the variables $y, z$ (see equation (1)). This will be further discussed, together with the numerical trials and results. 


\subsection{Hybrid Dynamic Programming}

In what follows the optimal treatment of a patient with a recently discovered infection, for instance with initial conditions near

$$
\begin{aligned}
& x_{0}=850 \text { cells } \mathrm{mm}^{-3}, y_{0}=41 \text { cells } \mathrm{mm}^{-3}, \\
& z_{0}=3760 \text { copies } \mathrm{ml}^{-1}, \lambda_{0}=5 \text { cells } \mathrm{mm}^{-3} \text { day }^{-1} \text {, }
\end{aligned}
$$

is illustrated. Dynamic Programming was implemented for a range of states around these initial conditions, covering expected behaviors of the patient under different medication strategies. The adopted discretization or spacing $(\Delta)$ of the variables and their corresponding lower $\left.{ }_{L}\right)$ and upper $\left.{ }_{U}\right)$ bounds are listed bellow:

$$
\begin{gathered}
\Delta x=50, \Delta y=10, \Delta z=500, \Delta \lambda=0.1, \Delta u_{1}=\Delta u_{2}=0.2, \\
x_{L}=500, y_{L}=1, z_{L}=10, \lambda_{L}=4, u_{1_{L}}=u_{2_{L}}=0, \\
x_{U}=1000, y_{U}=51, z_{U}=5010, \lambda_{U}=10, u_{1_{U}}=u_{2_{U}}=0.8
\end{gathered}
$$

It must be remarked that the lower thresholds $y_{L}$ and $z_{L}$ are given positive values. This is to avoid that, after an eventual rounding of their values, the discretized trajectories reach any point with $y=z=0$, since in that case the optimal strategy would continue with $u \equiv 0$ till the end, which is certainly erroneous (and dangerous). Indeed, the real system never reaches $y=z=$ $\lambda=0$ from an initial condition different from the unstable equilibrium, which means that any remaining infection $(y>0, z>0)$ will grow if $u=(0,0)$ and this growth should eventually be controlled with some $u_{i}>0$.

The upper limits $u_{1_{U}}=u_{2_{U}}=0.8$ adopted for the drug doses contemplate the protocols based on each pure component: the standard dosis for RTI alone is $* * * * * * * * * *$

The cost takes now a slightly different form

$$
\begin{aligned}
\mathcal{J}(u) & \triangleq \sum_{k=0}^{T / h^{t_{k+1}}} \int_{t_{k}}\left[a_{1} z(t)+m_{1} u_{1}(t)+m_{2} u_{2}(t)+a_{3}\left(\lambda(t)-\lambda_{m}\right)^{2}\right] d t+a_{4}(z(T / h)-\hat{z})^{2}, \\
t_{k} & \triangleq t_{0}+h k,
\end{aligned}
$$

where $x(t), y(t), z(t), \lambda(t)$ must be understood, in each interval $\left[t_{k} t_{k+1}\right)$, as the rounded result of the state-transition function $\phi\left(t, t_{0}, x, y, z, \lambda, u().\right)$ associated with the continuous-time model (1), namely

$$
\begin{aligned}
(x(t), y(t), z(t), \lambda(t))^{\prime} & =\phi\left(t, t_{k}, x_{k}, y_{k}, z_{k}, \lambda_{k}, \tilde{u}_{k}\right), \\
\left(x_{k+1}, y_{k+1}, z_{k+1}, \lambda_{k+1}\right)^{\prime} & =\operatorname{round}\left(\phi\left(t_{k+1}, t_{k}, x_{k}, y_{k}, z_{k}, \lambda_{k}, \tilde{u}_{k}\right)\right),
\end{aligned}
$$

the symbol $\tilde{u}_{k}$ being interpreted as the piecewise-constant control trajectory

$$
u(t)=\left(u_{1}(t), u_{1}(t)\right) \triangleq \tilde{u}_{k}(t) \equiv u_{k}=\left(u_{1_{k}}, u_{2_{k}}\right) \in \mathcal{U} \forall t \in\left[t_{k} t_{k+1}\right),
$$


and where 'round' acts over the values $\left(x\left(t_{k+1}\right), y\left(t_{k+1}\right), z\left(t_{k+1}\right), \lambda\left(t_{k+1}\right)\right)^{\prime}=$ $\phi\left(t_{k+1}, t_{k}, x_{k}, y_{k}, z_{k}, \lambda_{k}, \tilde{u}_{k}\right)$ in a 'safe' way, precisely

$$
\begin{aligned}
& x_{k+1} \triangleq \text { closest smaller value next to } x\left(t_{k+1}\right) \text { in } \mathcal{X} \\
& y_{k+1} \triangleq \text { closest bigger value next to } y\left(t_{k+1}\right) \text { in } \mathcal{Y} \\
& z_{k+1} \triangleq \text { closest bigger value next to } z\left(t_{k+1}\right) \text { in } \mathcal{Z} \\
& \lambda_{k+1} \triangleq \text { closest value next to } \lambda\left(t_{k+1}\right) \text { in } \mathcal{L}
\end{aligned}
$$

This means that, even when the time, state, and control spaces are discretized, the values of the states at a time $t_{k+1}$ are calculated by integrating the continuous-time dynamics of the control system (i.e. through the statetransition function $\phi$ of the system) by using the known states at time $t_{k}$ as initial conditions, and then rounding the states resulting from integration. That explains the adjective 'Hybrid' assigned to the Dynamic Programming technique used as numerical approach to the problem in this paper. The adopted value of $h=30$ days takes into consideration the observed 'peak time' (of approximately 20 days) occurring in the state variables and sensitivities (see [5]). The possibility of a hiding acute infection period is discarded this way, since at least the results of a blood analysis reflecting the situation will come at some intermediate point.

\section{$5 \quad$ Numerical results}

The first calculations made were for adult patients, from initial conditions as in Eq. (18), resulted in an optimal control strategy as shown in Fig. 4. The optimal doses of RTI stabilized at $0.6 \mathrm{~g}$ after the critical period of 180 days, while PI did the same at $0.4 \mathrm{~g}$. This was a good result is some aspects: first, both drugs were present in a nontrivial combination at all stages; and second, both of them stayed at a constant level from more than the last half of the period of consideration, which suggests that the medication $u_{\text {adults }}^{*}(t) \equiv(0.6,0.4)$ could become safe for approximately $t>180$ days, without need to repeat calculations for successive optimization periods. But the preference for RTIs over PIs was somewhat expected since $u_{1}$ acts by diminishing directly both the viral load and the thymus function dysfunctionality (and consequently lowering the costs weighted by $a_{1}, a_{3}$, and $a_{4}$ ), while increasing the drug cost weighted by $a_{2}$ at a lower rate than $u_{2}$ does (PIs are more expensive). This result is reinforced when the cost of thymus illness is not given preponderance in the composition of the cost, as was decided to be the case for adults.

Things change for children, as can be seen in Fig. 8, due to the different set of parameters used in calculations, as indicated in Eqns. $(7,16)$. The optimal medication for children stabilizes at $u_{\text {children }}^{*}(t) \equiv(0.4,0.6)$ for $t>180$ days, reversing the roles of RTI and PI in the optimal therapy for adults. This shows that an alternative combination of drugs, less agressive to the thymus functioning in children, can result optimal when the weights of the partial costs are tuned as to reflect a comprehensive evaluation of the situation. 


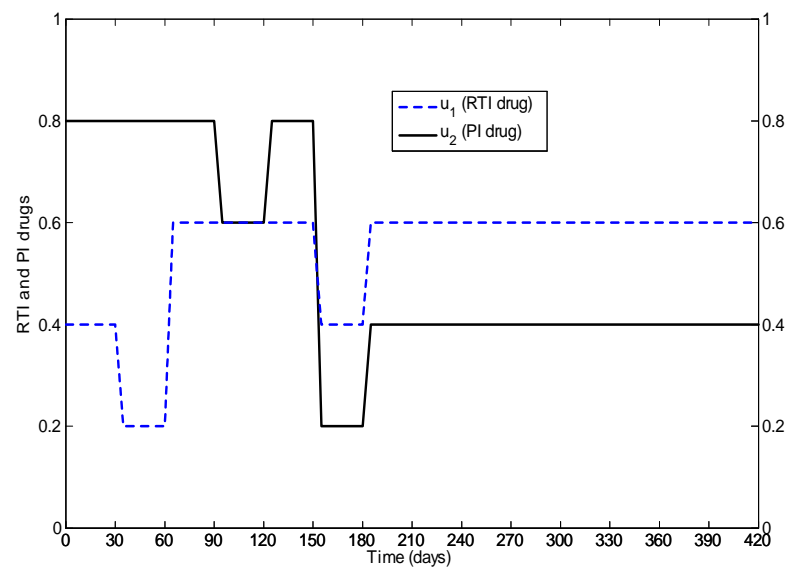

Figure 4: Optimal strategy (therapy) for adults, amongst all admissible combinations of RTI and PI drugs. Optimization horizon $T=420$ days.

\begin{tabular}{|c|c|c|c|c|c|}
\hline$a_{3}$ & $\int a_{1} z(t)$ & $\int a_{3}\left(\lambda-\bar{\lambda}_{m}\right)$ & $\int m_{1} u_{1} d t$ & $\int m_{2} u_{2} d t$ & $\begin{array}{c}\text { Cost of } \\
\text { medicatior }\end{array}$ \\
\hline \multirow{2}{*}{$\begin{array}{c}\text { children } \\
\text { adults }\end{array} 30$} & 476.30 & 16622 & $2495^{(\mathrm{C}}$ & $3889^{(0.4)}$ & 6383 \\
\hline & 416.81 & 8253 & $3142^{(0.6)}$ & $1814^{(0.2)}$ & 4956 \\
\hline $\begin{array}{c}\text { children } \\
\text { adults }\end{array} 100$ & $\begin{array}{l}393.72 \\
309.90\end{array}$ & $\begin{array}{l}53546 \\
25493\end{array}$ & $\begin{array}{l}2495^{(0.4)} \\
3788^{(0.6)}\end{array}$ & $\begin{array}{l}5184^{(0.6)} \\
3888^{(0.4)}\end{array}$ & $\begin{array}{l}7679 \\
7676\end{array}$ \\
\hline
\end{tabular}

Table 1: Cost values for children and adult treatments for $a_{3}=30,100 ; T=420$ days. The asymptotic optimal value of the drug $u_{i}$ appears as a superscript of the partial cost associated with such $u_{i}$.

The state trajectories for $x(t), z(t)$ and $\lambda(t)$, corresponding to the optimal control strategies, both dor adults and children, are depicted in Figs. 5-7. They show a very acceptable performance, judging from common-sense expectations for a good medication, i.e. it should quickly abate viral load while at the same time recuperates healthy cells and thymus function. For illustration purposes, and only in the adults' case, curves for the values of the states $x, z, \lambda$ that are generated in the intermediate calculations of the optimal control strategy by the Dynamic Programming scheme are added in Figs. 5-7. These values, obtained after applying the rounding conventions announced in Eqs. (26), are shown to be conservative, since the continuous-line curves (real behavior of the patient due to optimal medication) tend to the desired values more quickly and accurately than the remaining ones (Dynamic Programming rounded approximations).

Partial costs due to optimal medications in children and adult patients are 


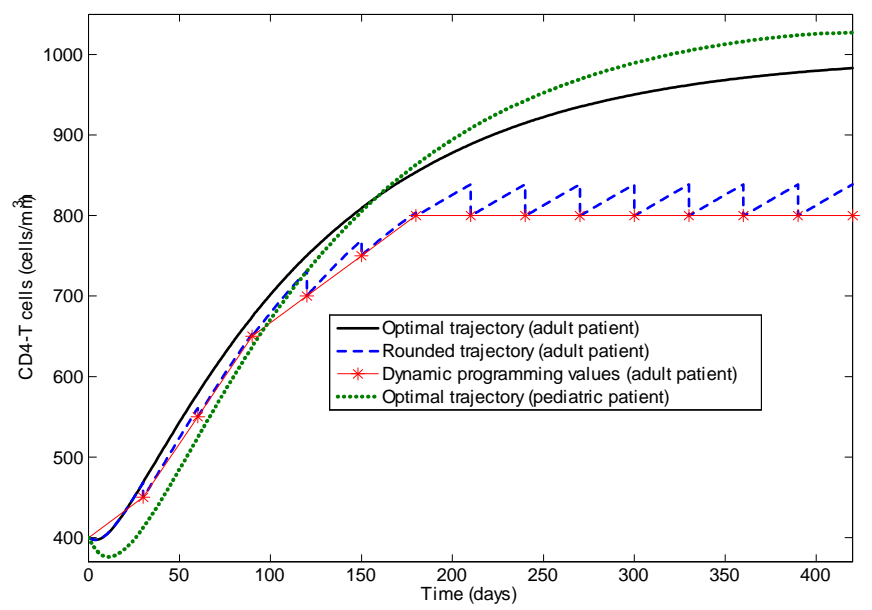

Figure 5: $x$-trajectories for children and adults resulting from their corresponding optimal medications. $x$-values used in Dynamic Programming calculations are also shown for adults.

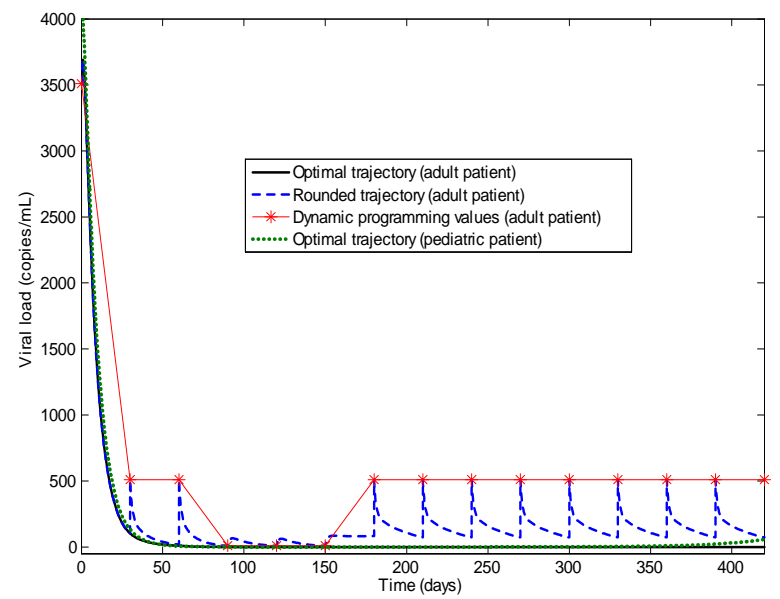

Figure 6: $z$-trajectory for children and adults resulting from their corresponding optimal medications. $z$-values used in Dynamic Programming calculations are also shown for adults. 


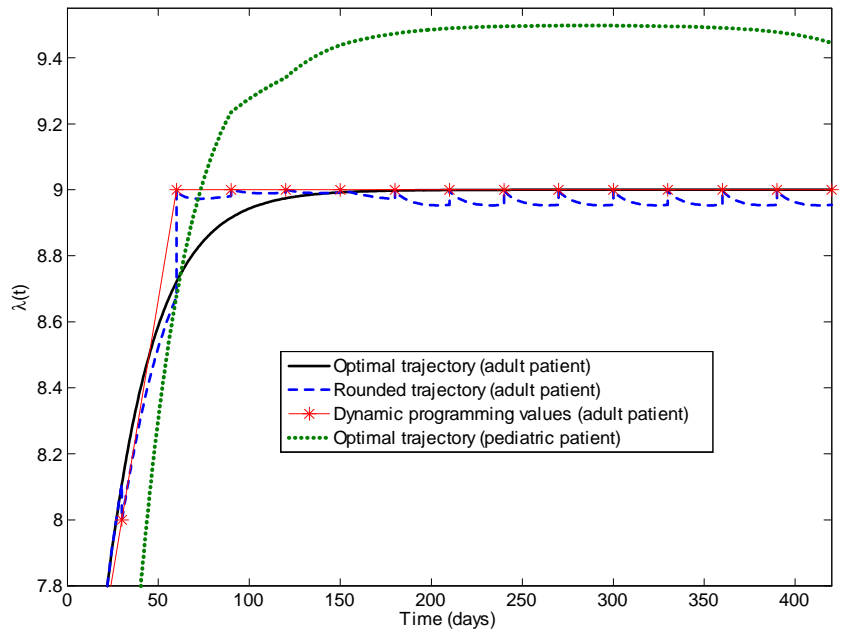

Figure 7: $\lambda$-trajectories for children and adults resulting from their corresponding optimal medications. $\lambda$-values used in Dynamic Programming calculations are also shown for adults.

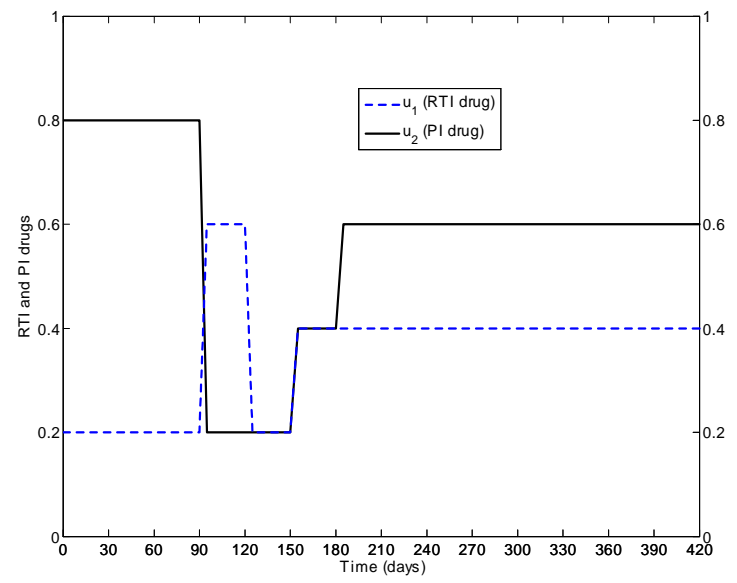

Figure 8: Optimal strategy (therapy) for children, amongst all admissible combinations of RTI and PI drugs. Optimization horizon $T=420$ days. 


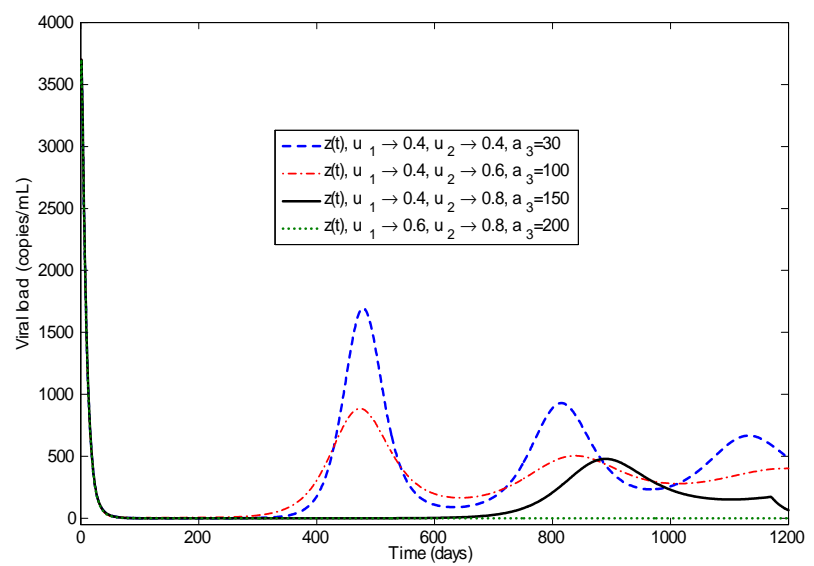

Figure 9: $z$-trajectory for children resulting from optimal combined RTI plus PI medications corresponding to different weights $a_{3}$ assigned to thymus' deficiency cost.

shown in Table 1 , for $a_{3}=30$ and $a_{3}=100$. In both cases the sum of the asymptotic optimal doses is the same for children and adults, although for $a_{3}=$ 100 such a sum is higher $\left(1 \mathrm{~g}\right.$ instead of $0.8 \mathrm{~g}$ for $\left.a_{3}=30\right)$, indicating that more concern for thymus function will require more drug all other objectives equally weighted. The cost of medication for children when $a_{3}=30$ is 1.28 times the cost for adults, but this is not too expensive to pay for as much as $16622 / 8253=2.0141$ times the all along deviation of the thymus function. For $a_{3}=100$ this argument is even stronger but the cost of medication rises both for children and adults to reach around 7680 dollars in 420 days. As $a_{3_{\text {chiildren }}}$ increases the recommended PI dosis increases and surpasses the one for RTIs. This could be rephrased by saying that emphasizing thymus concern in children patiens would amount to use increasing proportion of PI drugs replacing RTIs. This policy would be reinforced if the hypothesis of PI retarding the switching time from R5 a X4 virus [8] is confirmed by further empirical data.

For longer optimization horizons things become more complex. Figures 9, 10 depict respectively the $z$ - and $\lambda$-trajectories corresponding to the optimal medications calculated for children and a very long $T$ (=1200 days), using different values for $a_{3}$. The asymptotic recommended drug doses are higher than for shorter optimization periods, explained by the need to avoid significant rebounds in the viral load. This is an issue that deserve further study, above all considering that model parameters are no longer to be trusted to remain constant after such long periods of treatment, and the same can be expected of the partial cost weights. Perhaps long optimizarion horizons would require then nonautonomous formulations for both the dynamics and the cost function. 


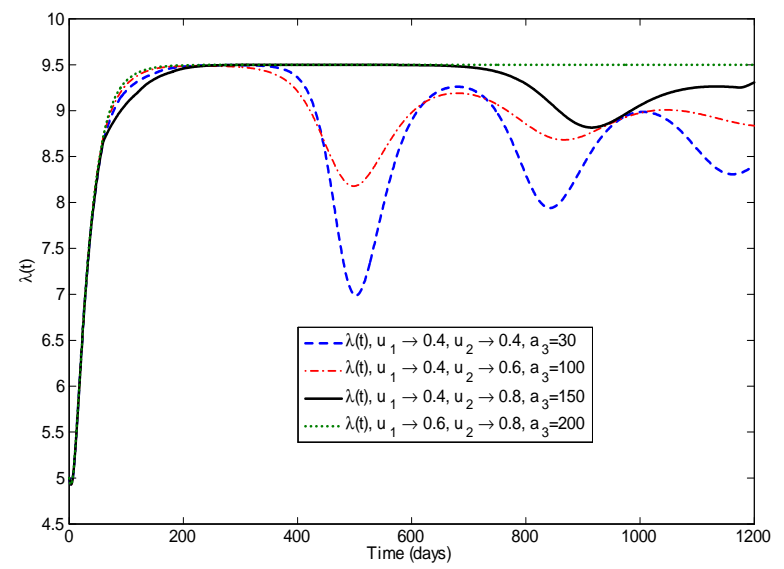

Figure 10: $\lambda$-trajectory for children resulting from optimal combined RTI plus PI medications corresponding to different weights $a_{3}$ assigned to thymus' deficiency cost.

\section{Conclusions}

A methodology, suitable for assessing drug combinations in HIV treatment of children and adult patients, has been presented. The problem was posed in the context of deterministic optimal control, although there are at present uncertainties concerning the dynamics of human thymus under viral infection and highly active medication than would probably require an stochastic setup in the future. It has been found that, providing the model be accurate enough, different drug combinations could result better than monotherapies, and that the best combinations are not completely obvious but discovered through mathematical analysis and numerical calculation. The choice for the dosis of two different drugs was to be determined every 30 days, and are to be taken constantly during the next period. The criteria for deciding what was best were a number of conflicting objectives as partial terms of a cost functional entailing evaluations during the whole optimization time-horizon and at the end of it. Classical Dynamic Programming proved to be useful, after proper adapting to the mixed continuous-discrete characteristics of the problem, in finding optimal medication strategies amongst so many degrees of freedom.

The problem had essentially two different setups: one for adult patients and another one for just-born children. Parameters for the dynamics and the evaluation functional of each setup were tuned to reflect existing knowledge, which is still little more than incipient. But results are promising: an inverse tendency in the proportion between the RTI and PI families of drugs seem to be recommendable for children in comparison with that for adults. These is to 
say: more PIs than RTIs for children, and the converse ratio for adults, when the concern for thymus function grows in appreciation with respct to other cost objectives. Another positive outcome is the stabilization of the composition of the optimal dosis after some 180 days towards a mix with significant PI share. This is regarded as 'milder' acceptable therapies, especially well received by children patients.

The variations of optimal medication strategies were also assessed when evaluating long periods, the increasing of the total amount of drugs being the expected consecuence of minimizing rebound risks for viral load. But it has to be recalled that no consideration of side-effects have entered the present methodology. Again, the longer the horizon under study, the greater the uncertainties are in the number and values of parameters and weight coefficients needed for a definitive problem formulation.

\section{References}

[1] P. Cahn, I. Cassetti, R. Wood, P. Phanuphak, L. Shiveley, R.C. Bethell, J. Sawyer, Efficacy and tolerability of 10-day monotherapy with apricitabine in antiretroviral-naive, HIV-1 infected patients, AIDS 20 (9) (2006) 12611268 .

[2] A. Cato, J. Qian, A. Hsu, B. Levy, J. Leonard, and R. Granneman, Multidose Pharmacokinetics of Ritonavir and Zidovudine in Human Immunodeficiency Virus-Infected Patients, Antimicrob. Agents Chemother. 42 (1998) 1788-1793.

[3] R. Cheynier, R. Bordi, M.L. Dion, R. Woods, J. Montaner, F. Harris, M. Lederman, R.P. Sekaly, Immunological improvement follonwing HAART coincides with restored thymic function. In: IAS Conference on HIV Pathogenesis and Treatment, Antivir. Ther. 8 (Suppl.1): abstract no. 15. Unite des Virus Lents, Institut Pasteur,Paris, France, 2003.

[4] R. Consolini, A. Legitimo, M. Milani, Primary thymic endocrine failure in HIV-1-infected children, Pathobiology 68(6) (2000) 251-7.

[5] V. Costanza, P.S. Rivadeneira, F.L. Biafore, C.E. D'Attellis, A Closed-Loop Approach to Antiretroviral Therapies for HIV Infection, Biomed. Signal Processing and Control 4 (2009) 139-148.

[6] V. Costanza, P.S. Rivadeneira, F.L. Biafore, C.E. D'Attellis, Taking Side Effects into Account for HIV Medication, IEEE Trans. Biomed. Eng. 57(9) (2010) 2079-2089.

[7] R. T. Davey, HIV-1 and T cell dynamics after interruption of highly active antiretroviral therapy (HAART) in patients with a history of sustained viral suppression, Proc. Nat. Acad. Sci. USA 98(26) (1999) 15109-15114. 
[8] P. Delobel, K. Sandres-Saune, M. Cazabat, C. Pasquier, B. Marchou, P. Massip, J. Izopet, R5 to X4 Switch of the Predominant HIV-1 Population in Cellular Reservoirs During Effective Highly Active Antiretroviral Therapy, J. Acquir. Immune Defic. Syndr. 38(4) (2005) 382-92.

[9] D. C. Douek, R. A. Koup, R. D. McFarland, J. L. Sullivan, K. Luzuriaga, Effect of HIV on thymic function before and after antiretroviral therapy in children. IN: 7th Conf. Retrovir. Oppor. Infect., San Franc. Calif. Jan 30-Feb 2, 7: 99 (2000).

[10] D. C. Douek, R. D. McFarland, P. H. Keiser, E. A. Gage, J. M. Massey, B. F. Haynes, M. A. Polis, A. T. Haase, M. B. Feinberg, J. L. Sullivan, B. D. Jamieson, J. A. Zack, L. J. Picker, R. A. Koup, Changes in thymic function with age and during the treatment of HIV infection, Nature 396(6712) (1998) 690-5.

[11] R .H. Fang, A. D. Colantonio, C. H. Uittenbogaart, The Role of the Thymus in HIV Infection: A 10-Year Perspective: The Thymus in Discordant Immunological and Virological Response, AIDS 22(2) (2008) 171-184.

[12] R. Hazra, C. Mackall, Thymic function in HIV infection, Curr. HIV/AIDS Rep. 2(1) (2005) 24-28.

[13] C. Hoffmann, B. Kamps, HIV Medicine. Flying Publisher, 2007. www.HIVmedicine.com

[14] D. E. Kirschner, R. Mehr, A. S. Perelson, Role of the Thymus in Pediatric HIV-1 Infection. JAIDS 18 (1998) 95-109.

[15] J. H. Ko, W. H. Kim, C. C. Chung, Optimized Structured Treatment Interruption for HIV Therapy and Its Performance Analysis on Controllability, IEEE Trans. Biomed. Eng. 53(3) (2006) 380-386.

[16] N. R. Manley, Mechanisms controlloing thymic homeostasis, involution, and rebound. Project Number: 1P01AI076514-01A1, 2009. http://projectreporter.nih.gov/project_info_details.cfm?aid=7569071

[17] M. J. Míguez-Burbano, M. Nair, J. E. Lewis, J. Fishman, The role of alcohol on platelets, thymus and cognitive performance among HIV-infected subjects: are they related?, Platelets 20(4) (2009) 260-7.

[18] M. A. Nowak MA, R. M. May, Pathogenesis-mathematical models of HIV and SIV infections, AIDS 7 (1993) 3-18.

[19] M. A. Nowak MA, R. M. May, Virus Dynamics: Mathematical Principles of Immunology and Virology. Oxford University Press, New York, 2000.

[20] P. Palumbo, H. Wu, E. Chadwick, P. Ruan, K. Luzuriaga, J. Rodman, R. Yogev, Virologic response to potent antiretroviral therapy and modeling of HIV dynamics in early pediatric infection, The Journal of infectious diseases 196(1) (2007) 23-9. 
[21] A. S. Perelson, D. E. Kirschner, R. de Boer, Dynamics of HIV infection of CD4+ T cells, Math. Biosci. 114 (1993) 81-125.

[22] A. S. Perelson, P. W. Nelson, Mathematical analysis of HIV-1 dynamics in vivo. SIAM Rev. 41 (1999) 3-44.

[23] A. S. Perelson, Modelling viral and immune system dynamics, Nature Reviews/Inmunology 2 (2002) 28-36.

[24] S. Resino, M. Leal, R. Correa, M. Genebat, V. Campos, M. A. MuñozFernández, Immune reconstitution in HIV-infected children on antiretroviral therapy, Inmunolgía, 24(1) (2005) 44-54.

[25] P. J. Ruane, G. J. Richmond, E. DeJesus, C. E. Hill-Zabala, S. C. Danehower, Q. Liao, J. Johnson, M. S. Shaefer, Pharmacodynamic effects of zidovudine $600 \mathrm{mg}$ once/day versus $300 \mathrm{mg}$ twice/day in therapy-naïve patients infected with human immunodeficiency virus, Pharmacotherapy 24(3) (2004) 307-312.

[26] A. Saitoh, K. K. Singh KK, S. Sandall, C. A. Powell, T. Fenton, C. V. Fletcher, K. Hsia, S. A. Spector, Association of CD4+ T-lymphocyte counts and new thymic emigrants in HIV-infected children during successful highly active antiretroviral therapy, J. Allergy Clin. Immunol. 117(4) (2006) 74852.

[27] M. F. Thomas, J. M. Vreeland, A socio-economic model for outcome assessment in health care management. In: Proceedings of the Academy of Accounting and Financial Studies, Volume 3, Number 2 Las Vegas, 1998.

[28] P. Ye, A. P. Kourtis, D. E. Kirschner, Reconstitution of thymic function in HIV-1 patients treated with highly active antiretroviral therapy, Clinical Immunology 106 (2003) 95-105. 